\title{
Sequence- and stereospecific assignment of methyl groups using paramagnetic lanthanides
}

\author{
Michael John, Christophe Schmitz, Ah Young Park, Nicholas E. Dixon, Thomas Huber, \\ and Gottfried Otting

\section{Supporting Information}

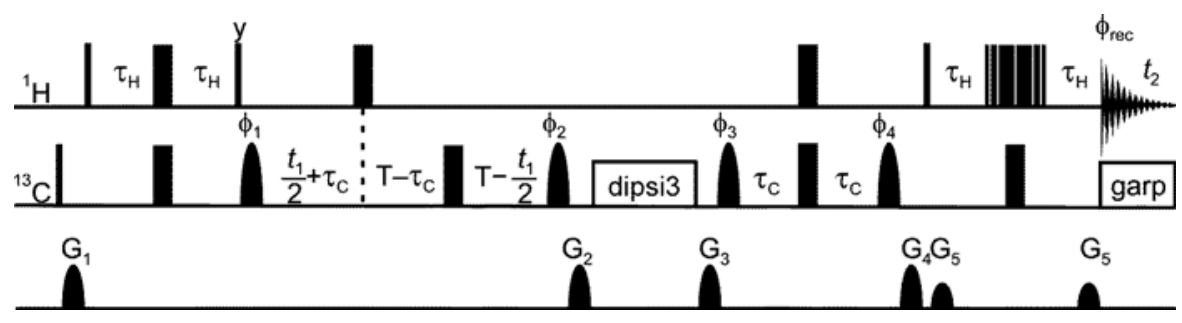

Figure S1. Pulse scheme of the 2D $(\mathrm{H}) \mathrm{C}(\mathrm{C}) \mathrm{H}-\mathrm{TOCSY}$ experiment used in this study. Parameters are as for the pulse schemes of Figure 1. Efficient magnetization transfer between the methyl groups of isopropyl groups was obtained by applying DIPSI3 mixing for 12 ms with a radiofrequency amplitude of $8.6 \mathrm{kHz}$. The Bruker pulse programs of this pulse sequence and of the pulse sequences of Figure 1 can be downloaded from http://rsc.anu.edu.au/ go/. 


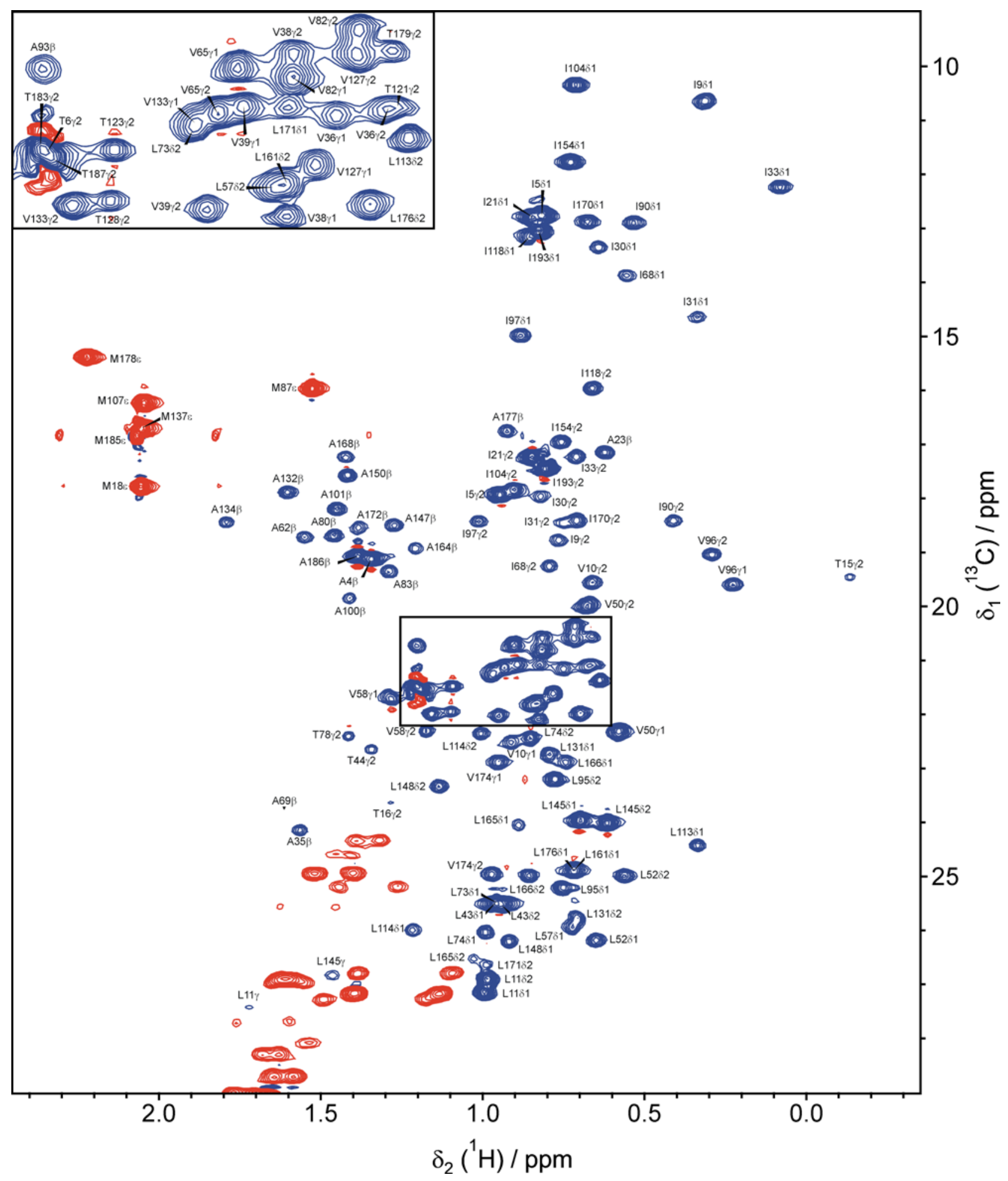

Figure S2. Assigned constant-time $(28 \mathrm{~ms}){ }^{13} \mathrm{C}-\mathrm{HSQC}$ spectrum of the cz- $\varepsilon 186 / \theta / \mathrm{La}^{3+}$ complex $\left({ }^{13} \mathrm{C} /{ }^{15} \mathrm{~N}\right.$ labeled cz- $\left.\varepsilon 186\right)$ at $\mathrm{pH} 7.2$ and $25{ }^{\circ} \mathrm{C}$. Only the region containing the methyl cross-peaks is shown. Cross-peaks from methyl groups of Val, Leu, Ile, Ala and Thr appear as positive peaks (blue), whereas cross-peaks from Met $\varepsilon \mathrm{CH}_{3}$ and all $\mathrm{CH}_{2}$ groups appear as negative peaks (red). 


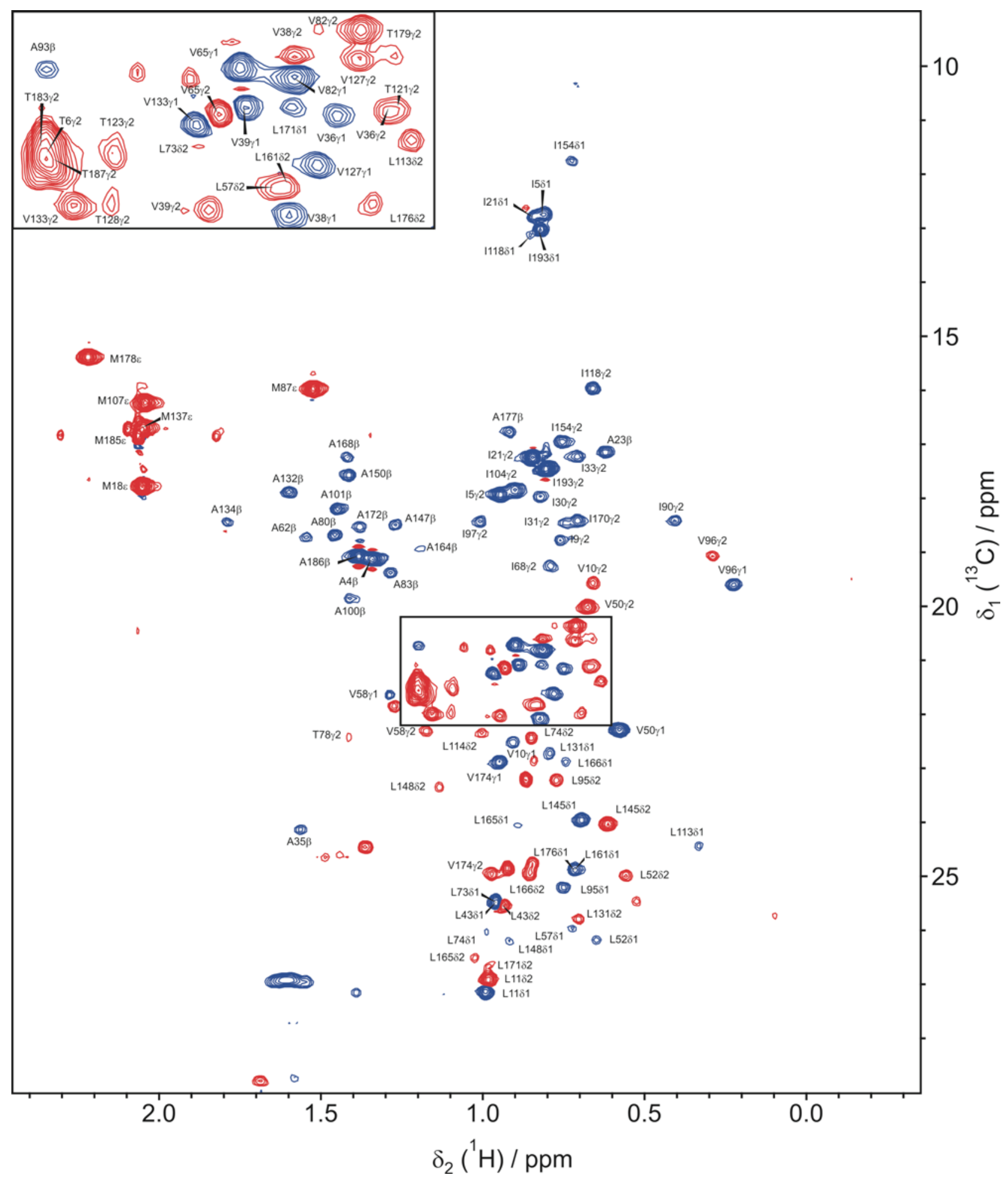

Figure S3. Assigned constant-time ${ }^{13} \mathrm{C}-\mathrm{HSQC}$ spectrum of the $\mathrm{cz}-\varepsilon 186 / \theta / \mathrm{La}^{3+}$ complex, where cz$\varepsilon 186$ was biosynthetically fractionally ${ }^{13} \mathrm{C}$-labeled using $20 \%$ uniformly ${ }^{13} \mathrm{C}$-labeled glucose. Parameters and plot region as in Figure S2. Cross-peaks from Val $\gamma 1$, Leu $\delta 1$, and Ala $\beta$ methyl groups are positive (blue). Cross-peaks from Val $\gamma 2$, Leu $\delta 2$, Thr $\gamma 2$ and Met $\varepsilon$ methyl groups are negative (red). Cross-peaks from Ile $\delta 1$ and $\gamma 2$ methyl groups are mostly invisible due to scrambling of ${ }^{13} \mathrm{C}$ during Ile biosynthesis. 


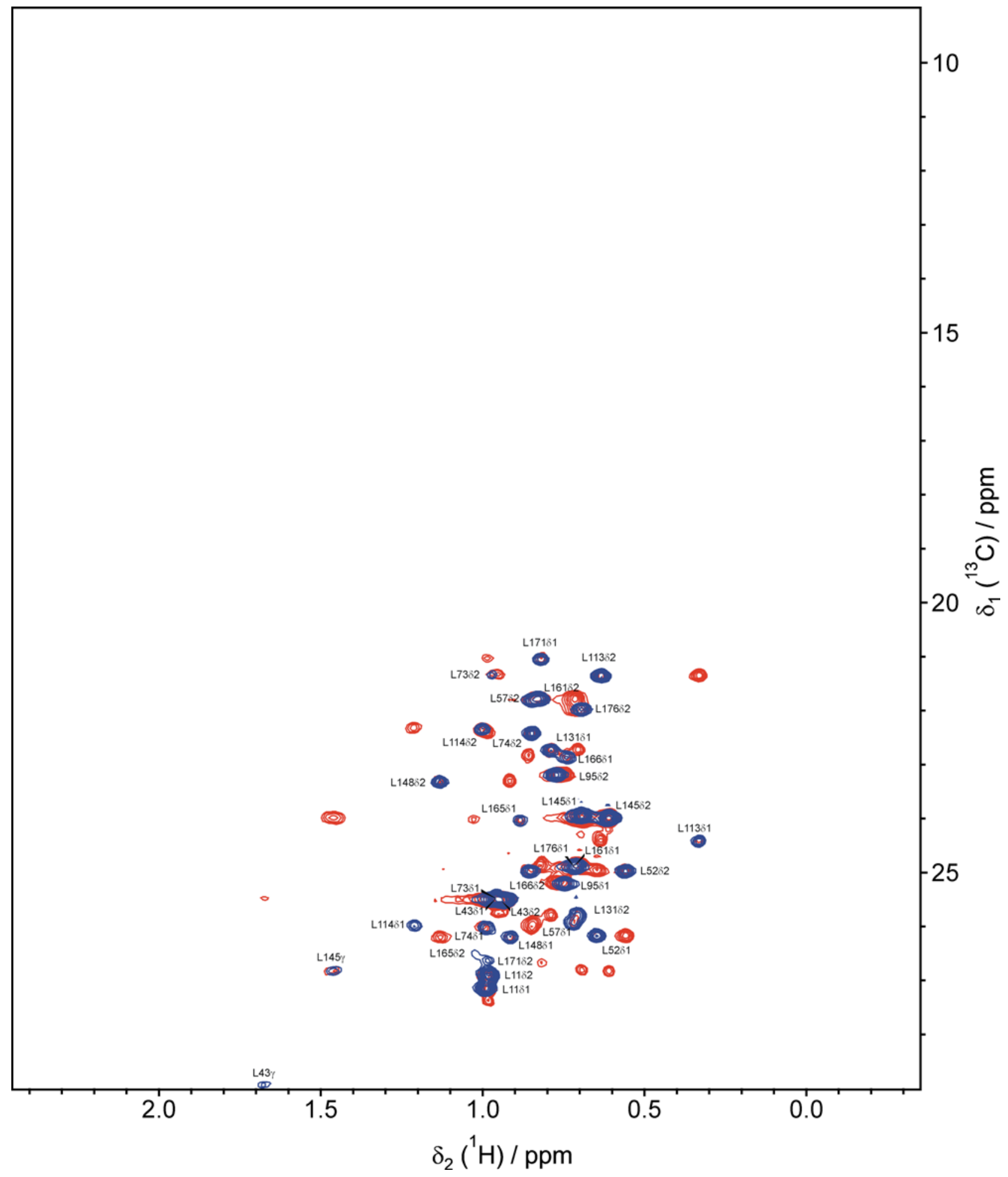

Figure S4. Assigned constant-time ${ }^{13} \mathrm{C}-\mathrm{HSQC}$ spectrum of the $\mathrm{cz}-\varepsilon 186 / \theta / \mathrm{La}^{3+}$ complex containing ${ }^{13} \mathrm{C} /{ }^{15} \mathrm{~N}$-Leu labeled cz- $\varepsilon 186$ (blue) superimposed onto a 2D $(\mathrm{H}) \mathrm{C}(\mathrm{C}) \mathrm{H}-\mathrm{TOCSY}$ spectrum of the same sample (red). The assignments of the ${ }^{13} \mathrm{C}$-HSQC cross-peaks are indicated. The three mobile residues Leu11, Leu43 and Leu145 also show one-bond correlations between $\delta_{\mathrm{CH}}$ and $\gamma \mathrm{CH}$ groups. 
Figure S5. Comparisons of calculated and experimental PCS in the cz- $186 / \theta / \mathrm{Dy}^{3+}$ complex for methyl groups of (a) Met, (b) Ala, (c) Thr, (d) Val, (e) Leu, and (f) Ile. ${ }^{13} \mathrm{C}$ and ${ }^{1} \mathrm{H}$ PCS are plotted with filled and open bars, respectively, in the sequence $\mathrm{C}^{\gamma 1} / \mathrm{H}^{\gamma 1} / \mathrm{C}^{\gamma 2} / \mathrm{H}^{\gamma 2}(\mathrm{Val}), \mathrm{C}^{\delta 1} / \mathrm{H}^{\delta 1} / \mathrm{C}^{\delta 2} / \mathrm{H}^{\delta 2}$ (Leu), and $\mathrm{C}^{\gamma 2} / \mathrm{H}^{\gamma 2} / \mathrm{C}^{\delta 1} / \mathrm{H}^{\delta 1}$ (Ile). The distances $r_{\mathrm{C}-\mathrm{Ln}}$ are indicated in $\AA$ at the top of each plot. For residues with two methyl groups, the distance value shown at the top refers to the $\mathrm{C}^{\gamma 1}$ (Val), $\mathrm{C}^{\delta 1}$ (Leu), or $\mathrm{C}^{\delta 1}$ (Ile) atom.
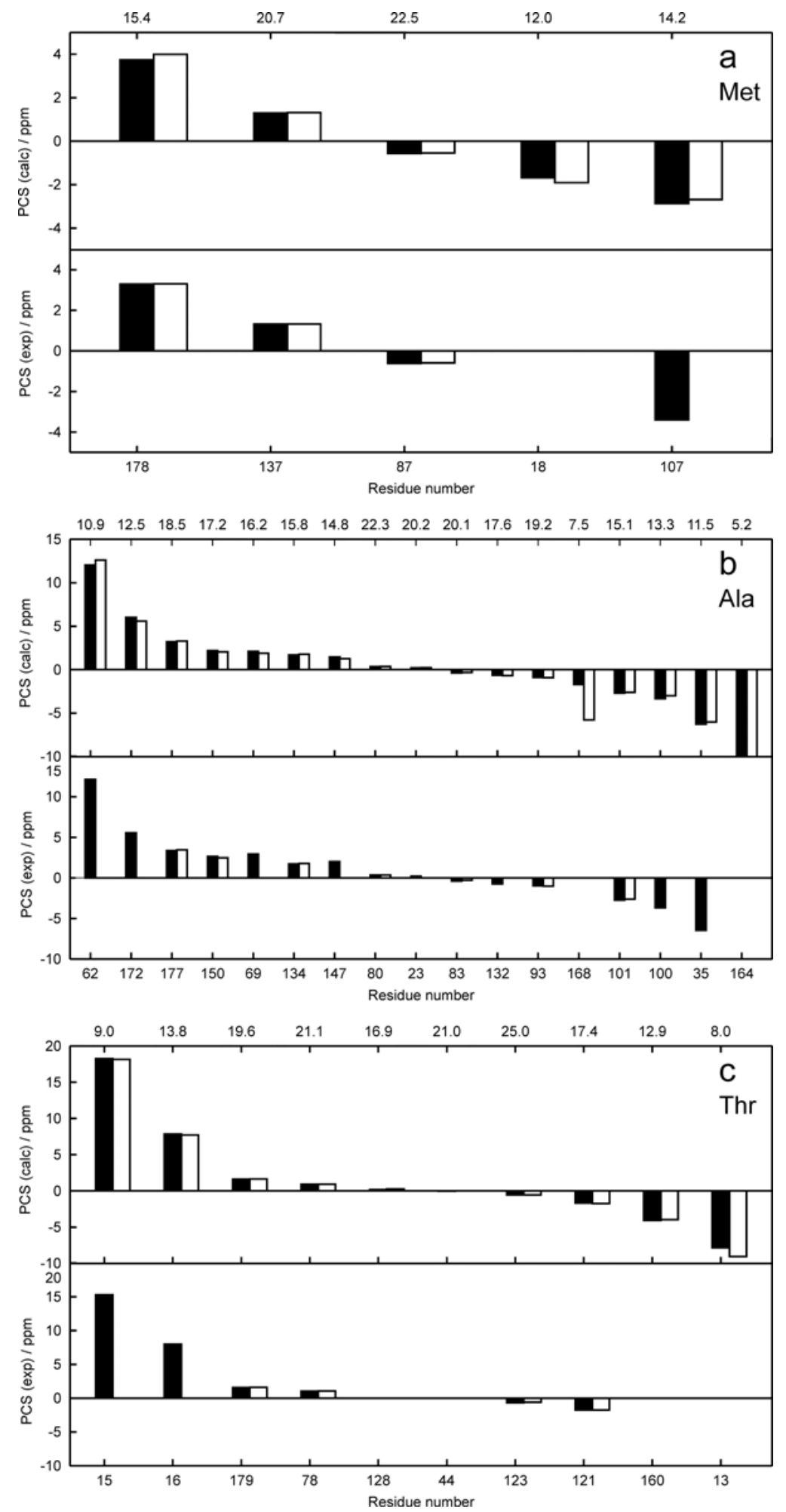
Figure S5 continued
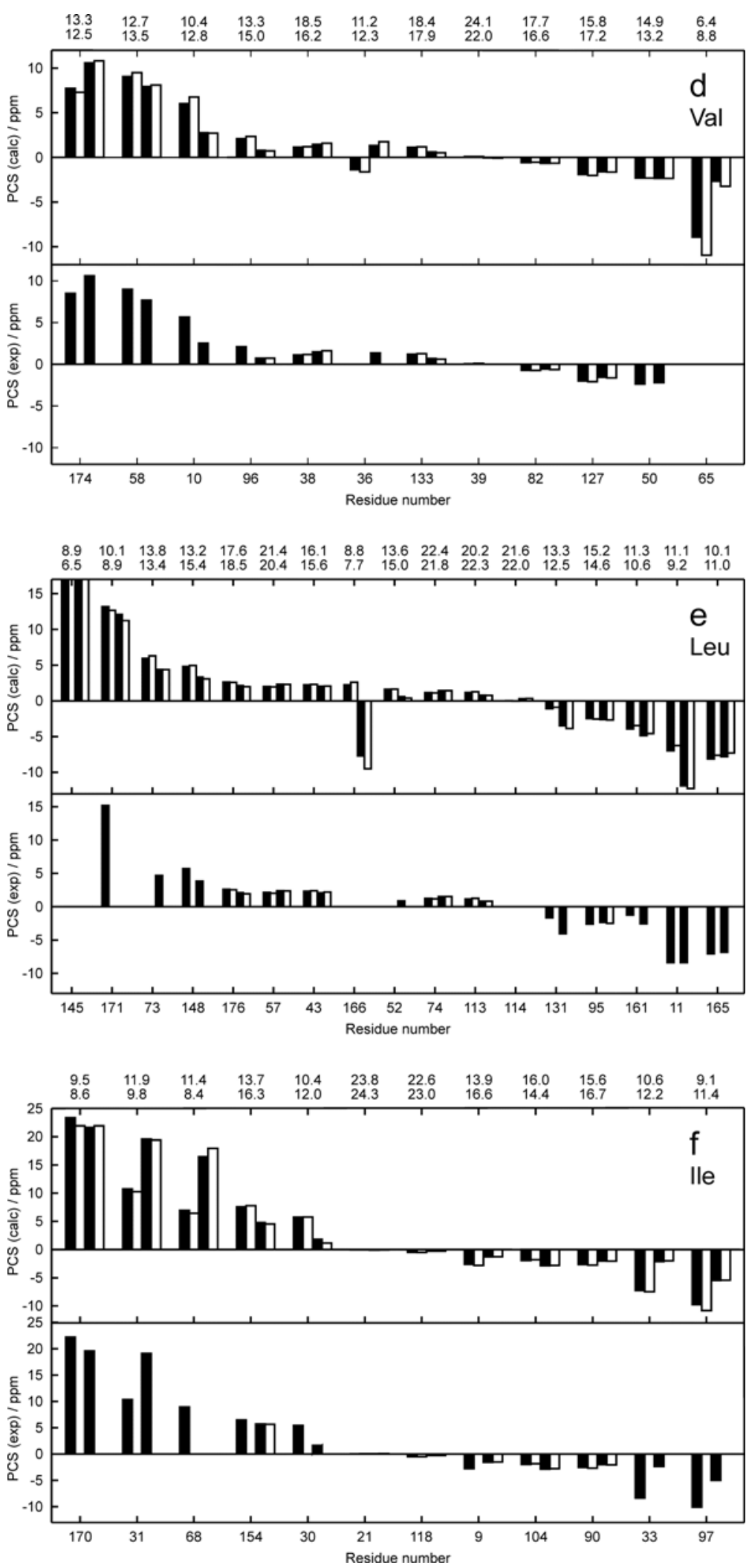
Figure S6. Comparisons of calculated and experimental ${ }^{13} \mathrm{C}$ and ${ }^{1} \mathrm{H}$ PCS as in Figure S5, but for the $\mathrm{cz}-\varepsilon 186 / \theta / \mathrm{Yb}^{3+}$ complex.
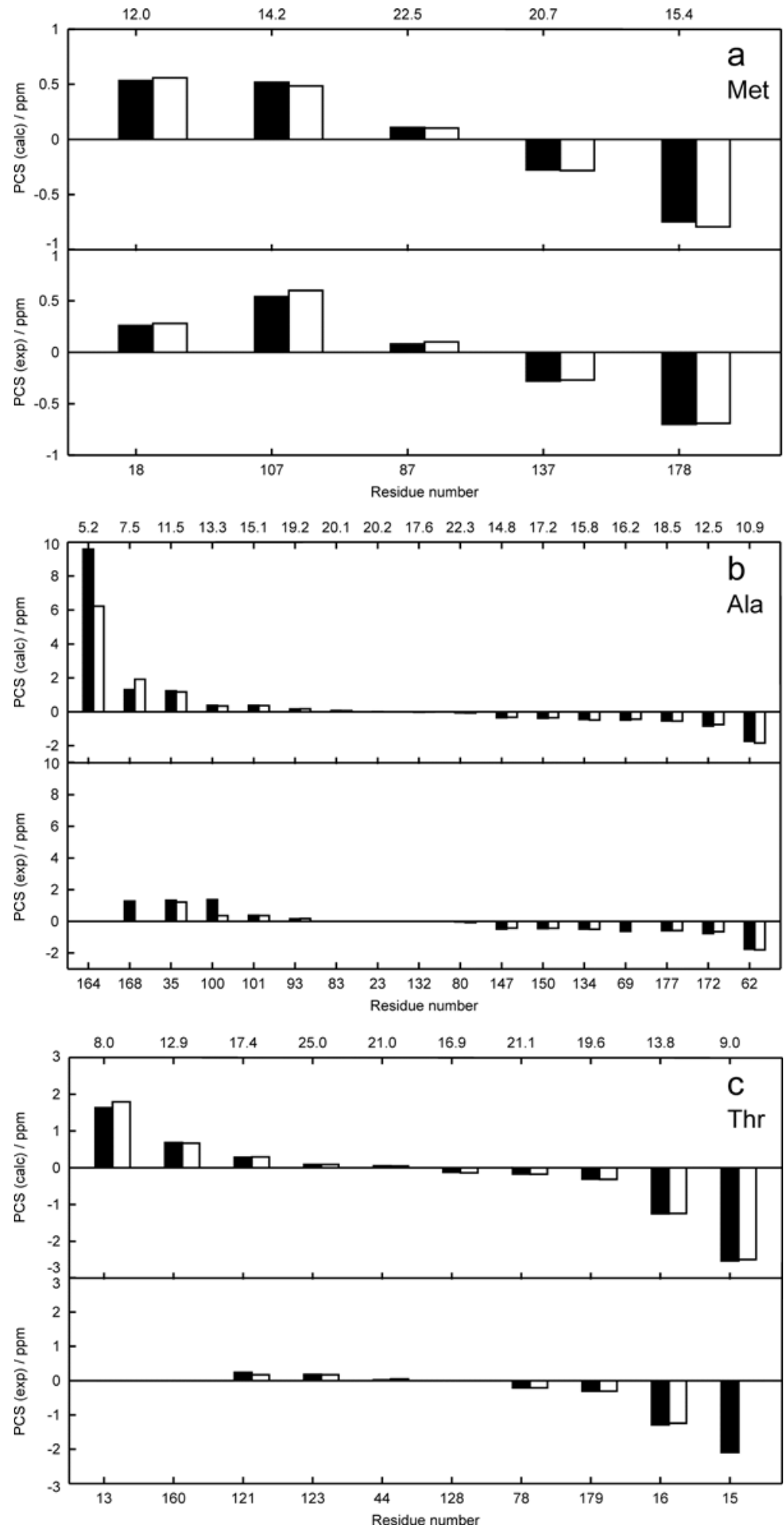
Figure S6 continued
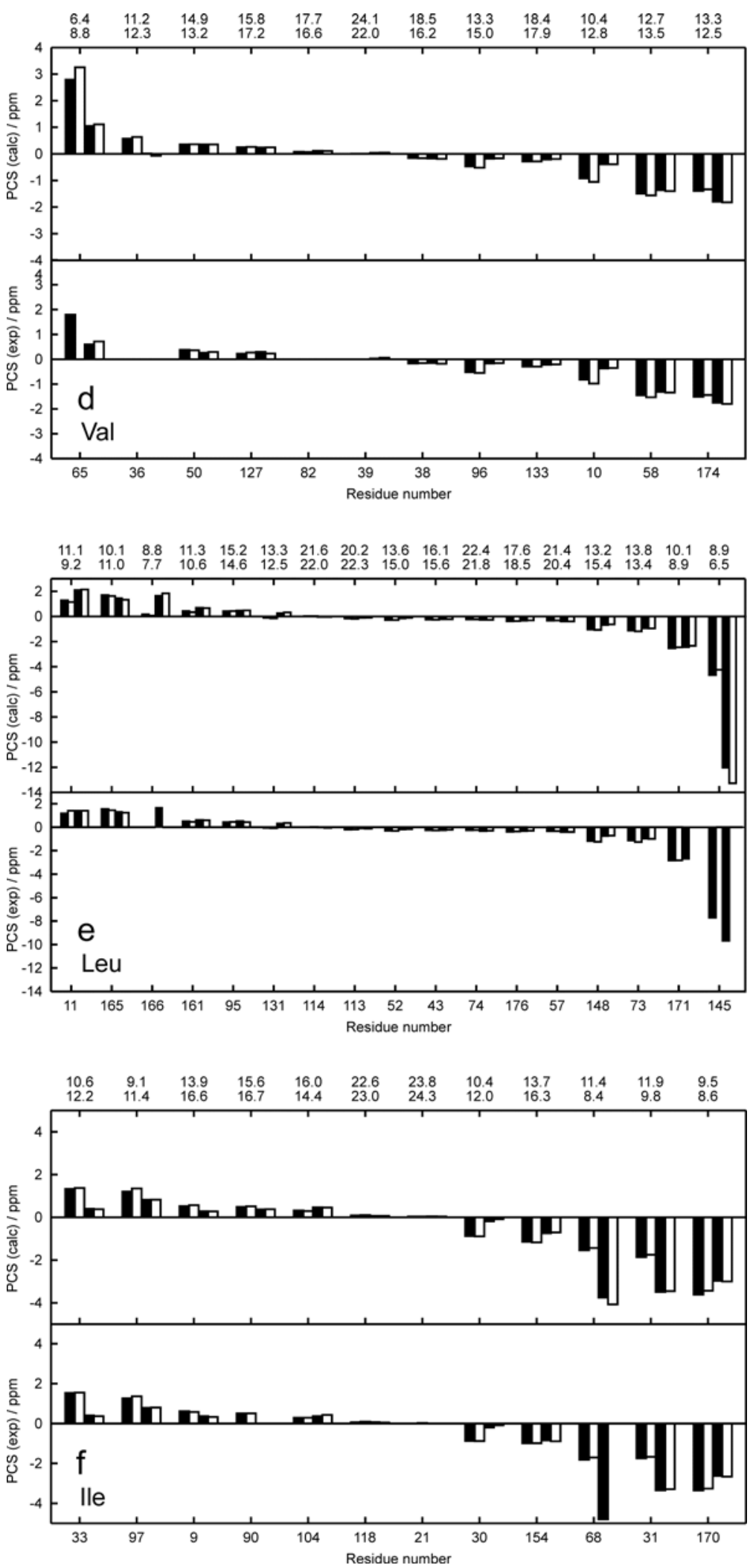
Table S1. ${ }^{13} \mathrm{C}$ and ${ }^{1} \mathrm{H}$ chemical shifts (ppm) of methyl groups of cz- $\varepsilon 186$ in the cz- $\varepsilon 186 / \theta / \mathrm{Ln}^{3+}$ complexes used in this study. ${ }^{\mathrm{a}}$

\begin{tabular}{|c|c|c|c|c|c|c|c|c|}
\hline \multicolumn{2}{|l|}{ Group } & \multirow[t]{2}{*}{$\begin{array}{l}r_{\mathrm{C}-\mathrm{Ln}} \\
(\AA)\end{array}$} & \multicolumn{2}{|c|}{$\begin{array}{l}\mathrm{Cz}-\varepsilon 186 / \theta / \mathrm{La}^{3+} \\
{ }^{13} \mathrm{C}{ }^{1} \mathrm{H}\end{array}$} & \multicolumn{2}{|c|}{$\begin{array}{c}\mathrm{Cz}-\varepsilon 186 / \theta / \mathrm{Dy}^{3+} \\
{ }^{13} \mathrm{C}{ }^{1}{ }^{1} \mathrm{H}\end{array}$} & \multicolumn{2}{|c|}{$\mathrm{CZ}-\varepsilon 186 / \theta / \mathrm{Yb}^{3+}$} \\
\hline \multicolumn{8}{|c|}{ Methionine } & \\
\hline M1 8 & $\varepsilon$ & 12.0 & 17.78 & 2.06 & & & 18.04 & 2.34 \\
\hline M8 7 & $\varepsilon$ & 22.5 & 15.97 & 1.53 & 15.32 & 0.93 & 16.05 & 1.63 \\
\hline M107 & $\varepsilon$ & 14.2 & 16.23 & 2.04 & 12.84 & & 16.77 & 2.64 \\
\hline M137 & $\varepsilon$ & 20.7 & 16.69 & 2.05 & 18.00 & 3.38 & 16.41 & 1.78 \\
\hline M178 & $\varepsilon$ & 15.4 & 15.39 & 2.22 & 18.67 & 5.53 & 14.69 & 1.53 \\
\hline M185 & $\varepsilon$ & & 16.83 & 2.07 & 17.32 & 2.55 & 16.75 & 1.98 \\
\hline \multicolumn{9}{|c|}{ Alanine } \\
\hline A4 & $\beta$ & & 19.14 & 1.35 & & & & \\
\hline A23 & $\beta$ & 20.2 & 17.15 & 0.62 & 17.35 & & & \\
\hline A35 & $\beta$ & 11.5 & 24.14 & 1.57 & 17.70 & & 25.47 & 2.79 \\
\hline A 62 & $\beta$ & 10.9 & 18.72 & 1.55 & 30.88 & & 16.97 & -0.24 \\
\hline A 69 & $\beta$ & 16.2 & 23.77 & 1.61 & 26.74 & & 23.15 & \\
\hline A8 0 & $\beta$ & 22.3 & 18.68 & 1.46 & 19.04 & 1.84 & 18.66 & 1.39 \\
\hline A83 & $\beta$ & 20.1 & 19.35 & 1.29 & 18.97 & 1.00 & & \\
\hline A93 & $\beta$ & 19.2 & 20.74 & 1.21 & 19.78 & 0.22 & 20.90 & 1.39 \\
\hline A100 & $\beta$ & 13.3 & 19.85 & 1.41 & 16.19 & & 21.22 & 1.77 \\
\hline A101 & $\beta$ & 15.1 & 18.20 & 1.45 & 15.48 & -1.16 & 18.58 & 1.82 \\
\hline A132 & $\beta$ & 17.6 & 17.89 & 1.60 & 17.16 & & & \\
\hline A134 & $\beta$ & 15.8 & 18.44 & 1.79 & 20.16 & 3.58 & 17.96 & 1.30 \\
\hline A147 & $\beta$ & 14.8 & 18.49 & 1.28 & 20.54 & & 18.01 & 0.86 \\
\hline A150 & $\beta$ & 17.2 & 17.57 & 1.42 & 20.22 & 3.91 & 17.12 & 0.99 \\
\hline A164 & $\beta$ & 5.2 & 18.93 & 1.21 & & & & \\
\hline A168 & $\beta$ & 7.5 & 17.24 & 1.42 & & & 18.52 & \\
\hline A172 & $\beta$ & 12.5 & 18.54 & 1.38 & 24.10 & & 17.77 & 0.73 \\
\hline A177 & $\beta$ & 18.5 & 16.75 & 0.92 & 20.11 & 4.39 & 16.17 & 0.34 \\
\hline A186 & $\beta$ & & 19.07 & 1.39 & 19.45 & 1.77 & 19.02 & 1.32 \\
\hline \multicolumn{9}{|c|}{ Threonine } \\
\hline \multicolumn{9}{|c|}{ T3 $\gamma 2$} \\
\hline $\mathrm{T} 6$ & $\gamma 2$ & & 21.50 & 1.20 & 21.53 & 1.25 & & \\
\hline T13 & $\gamma 2$ & 8.0 & & & & & & \\
\hline T15 & $\gamma 2$ & 9.0 & 19.45 & -0.13 & 34.77 & & 17.36 & \\
\hline $\mathrm{T} 16$ & $\gamma 2$ & 13.8 & 23.64 & 1.28 & 31.65 & & 22.35 & 0.04 \\
\hline $\mathrm{T} 44$ & $\gamma^{2}$ & 21.0 & 22.65 & 1.34 & 22.64 & 1.35 & 22.67 & 1.39 \\
\hline T78 & $\gamma^{2}$ & 21.1 & 22.40 & 1.42 & 23.44 & 2.48 & 22.19 & 1.21 \\
\hline T121 & $\gamma_{2}$ & 17.4 & 21.07 & 0.66 & 19.34 & -1.07 & 21.31 & 0.83 \\
\hline T123 & $\gamma 2$ & 25.0 & 21.48 & 1.09 & 20.80 & 0.50 & 21.66 & 1.26 \\
\hline T128 & $\gamma 2$ & 16.9 & 21.93 & 1.10 & & & & \\
\hline T160 & $\gamma^{2}$ & 12.9 & & & & & & \\
\hline T179 & $\gamma 2$ & 19.6 & 20.56 & 0.66 & 22.15 & 2.28 & 20.25 & 0.35 \\
\hline T183 & $\gamma^{2}$ & & 21.44 & 1.21 & 22.16 & 1.91 & 21.33 & 1.08 \\
\hline T187 & $\gamma^{2}$ & & 21.57 & 1.19 & 21.88 & 1.51 & 21.50 & 1.13 \\
\hline \multicolumn{9}{|l|}{ Valine } \\
\hline \multirow{2}{*}{ V10 $\gamma$} & $\gamma 1$ & 10.4 & 22.52 & 0.91 & 28.21 & & 21.70 & -0.07 \\
\hline & $\gamma^{2}$ & 12.8 & 19.56 & 0.66 & 22.13 & & 19.19 & 0.31 \\
\hline \multirow{2}{*}{ V36 } & $\gamma 1$ & 11.2 & 21.16 & 0.75 & & & & \\
\hline & $\gamma^{2}$ & 12.3 & 21.09 & 0.67 & 21.97 & & & \\
\hline \multirow[t]{2}{*}{ V38 } & $\gamma 1$ & 18.5 & 22.09 & 0.83 & 23.20 & 2.03 & 21.92 & 0.67 \\
\hline & $\gamma_{2}$ & 16.2 & 20.58 & 0.82 & 22.08 & 2.42 & 20.43 & 0.64 \\
\hline
\end{tabular}




\begin{tabular}{|c|c|c|c|c|c|c|c|c|}
\hline \multirow[t]{2}{*}{ V39 } & $\gamma 1$ & 24.1 & 21.08 & 0.89 & 21.14 & 1.00 & & \\
\hline & $\gamma^{2}$ & 22.0 & 22.03 & 0.95 & & & 22.06 & 1.01 \\
\hline \multirow[t]{2}{*}{ V50 } & $\gamma 1$ & 14.9 & 22.32 & 0.58 & 19.95 & & 22.70 & 0.94 \\
\hline & $\gamma 2$ & 13.2 & 20.00 & 0.68 & 17.80 & & 20.26 & 0.97 \\
\hline \multirow[t]{2}{*}{ V58 } & $\gamma 1$ & 12.7 & 21.64 & 1.29 & 30.67 & & 20.19 & -0.24 \\
\hline & $\gamma 2$ & 13.5 & 22.31 & 1.18 & 30.02 & & 21.00 & -0.16 \\
\hline \multirow[t]{2}{*}{ V65 } & $\gamma 1$ & 6.4 & 20.72 & 0.90 & & & 22.51 & \\
\hline & $\gamma 2$ & 8.8 & 21.14 & 0.93 & & & 21.74 & 1.65 \\
\hline \multirow[t]{2}{*}{ V82 } & $\gamma 1$ & 17.7 & 20.81 & 0.82 & 20.09 & 0.08 & & \\
\hline & $\gamma 2$ & 16.6 & 20.37 & 0.72 & 19.79 & 0.10 & & \\
\hline \multirow[t]{2}{*}{ V96 } & $\gamma 1$ & 13.3 & 19.60 & 0.23 & 21.73 & & 19.08 & -0.32 \\
\hline & $\gamma^{2}$ & 15.0 & 19.04 & 0.29 & 19.83 & 1.04 & 18.87 & 0.13 \\
\hline \multirow[t]{2}{*}{ V127 } & $\gamma 1$ & 15.8 & 21.62 & 0.78 & 19.64 & $-1 \cdot 32$ & 21.84 & 1.05 \\
\hline & $\gamma 2$ & 17.2 & 20.59 & 0.72 & 19.02 & -0.93 & 20.89 & 0.95 \\
\hline \multirow[t]{2}{*}{ V133 } & $\gamma 1$ & 17.9 & 21.22 & 0.97 & 22.43 & 2.24 & 20.92 & 0.67 \\
\hline & $\gamma 2$ & 18.4 & 21.99 & 1.16 & 22.69 & 1.76 & 21.77 & 0.95 \\
\hline \multirow[t]{2}{*}{ V1 74} & $\gamma 1$ & 13.3 & 22.88 & 0.95 & 31.40 & & 21.37 & -0.49 \\
\hline & $\gamma 2$ & 12.5 & 24.96 & 0.97 & 35.55 & & 23.21 & -0.83 \\
\hline \multicolumn{9}{|c|}{ Leucine } \\
\hline \multirow[t]{2}{*}{ L11 } & $\delta 1$ & 11.1 & 27.14 & 1.00 & 18.89 & & 28.30 & 2.40 \\
\hline & $\delta 2$ & 9.2 & 26.90 & 0.99 & 18.65 & & 28.30 & 2.39 \\
\hline \multirow[t]{2}{*}{ L4 3} & $\delta 1$ & 16.1 & 25.49 & 0.96 & 27.78 & 3.35 & 25.24 & 0.69 \\
\hline & $\delta 2$ & 15.6 & 25.52 & 0.94 & 27.63 & 3.15 & 25.26 & 0.72 \\
\hline \multirow[t]{2}{*}{ L52 } & $\delta 1$ & 13.6 & 26.18 & 0.65 & & & 25.88 & 0.34 \\
\hline & $\delta 2$ & 15.0 & 24.99 & 0.56 & 25.93 & & 24.81 & 0.39 \\
\hline \multirow[t]{2}{*}{ L5 7} & $\delta 1$ & 21.4 & 25.94 & 0.72 & 28.07 & 2.74 & 25.61 & 0.39 \\
\hline & $\delta 2$ & 20.4 & 21.82 & 0.85 & 24.23 & 3.21 & 21.40 & 0.44 \\
\hline \multirow[t]{2}{*}{ L 73} & $\delta 1$ & 13.8 & 25.49 & 0.96 & & & 24.36 & -0.30 \\
\hline & $\delta 2$ & 13.4 & 21.30 & 0.97 & 25.98 & & 20.34 & -0.02 \\
\hline \multirow[t]{2}{*}{ L 74} & $\delta 1$ & 22.4 & 26.03 & 0.99 & 27.27 & 2.17 & 25.78 & 0.75 \\
\hline & $\delta 2$ & 21.8 & 22.44 & 0.85 & 23.95 & 2.39 & 22.12 & 0.55 \\
\hline L95 & $\delta 1$ & 15.2 & 25.21 & 0.75 & 22.60 & & 25.64 & 1.20 \\
\hline & $\delta 2$ & 14.6 & 23.20 & 0.78 & 20.84 & -1.73 & 23.72 & 1.21 \\
\hline L113 & $\delta 1$ & 20.2 & 24.42 & 0.34 & 25.63 & 1.59 & 24.22 & 0.15 \\
\hline & $\delta 2$ & 22.3 & 21.37 & 0.64 & 22.17 & 1.45 & 21.27 & 0.53 \\
\hline L114 & $\delta 1$ & 21.6 & 26.00 & 1.22 & & & 26.00 & 1.24 \\
\hline & $\delta 2$ & 22.0 & 22.35 & 1.01 & & & 22.33 & 0.95 \\
\hline L131 & $\delta 1$ & 13.3 & 22.74 & 0.79 & 21.14 & & 22.69 & 0.70 \\
\hline & $\delta 2$ & 12.5 & 25.78 & 0.71 & 21.89 & & 26.09 & 1.07 \\
\hline L145 & $\delta 1$ & 8.9 & 23.96 & 0.70 & & & 16.25 & \\
\hline & $\delta 2$ & 6.5 & 24.01 & 0.62 & & & 14.34 & \\
\hline L148 & $\delta 1$ & 13.2 & 26.21 & 0.92 & 31.96 & & 25.03 & -0.32 \\
\hline & $\delta 2$ & 15.4 & 23.33 & 1.14 & 27.21 & & 22.59 & 0.42 \\
\hline L161 & $\delta 1$ & 11.3 & 24.89 & 0.72 & 23.66 & & 25.39 & 1.18 \\
\hline & $\delta 2$ & 10.6 & 21.78 & 0.83 & 19.26 & & 22.39 & 1.42 \\
\hline L165 & $\delta 1$ & 10.1 & 24.05 & 0.89 & 17.02 & & 25.60 & 2.34 \\
\hline & $\delta 2$ & 11.0 & 26.53 & 1.03 & 19.74 & & 27.83 & 2.26 \\
\hline L1 66 & $\delta 1$ & 8.8 & 22.89 & 0.74 & & & & \\
\hline & $\delta 2$ & 7.7 & 24.99 & 0.86 & & & 26.66 & \\
\hline L171 & $\delta 1$ & 10.1 & 21.06 & 0.82 & 36.23 & & 18.23 & -2.00 \\
\hline & $\delta 2$ & 8.9 & 26.65 & 0.99 & & & 23.97 & \\
\hline L176 & $\delta 1$ & 17.6 & 24.89 & 0.72 & 27.48 & 3.25 & 24.51 & 0.36 \\
\hline & $\delta 2$ & 18.5 & 21.98 & 0.70 & 24.08 & 2.64 & 21.66 & 0.40 \\
\hline Isole & euc: & & & & & & & \\
\hline I 5 & $\gamma 2$ & & 17.94 & 0.95 & 17.86 & 0.87 & 17.96 & 0.97 \\
\hline & $\delta 1$ & & 12.76 & 0.82 & 12.66 & 0.71 & & \\
\hline I 9 & $\gamma 2$ & 13.9 & 18.78 & 0.77 & 16.02 & & 19.39 & 1.35 \\
\hline & $\delta 1$ & 16.6 & 10.64 & 0.31 & 9.09 & -1.20 & 11.00 & 0.64 \\
\hline
\end{tabular}




\begin{tabular}{|c|c|c|c|c|c|c|c|c|}
\hline \multirow[t]{2}{*}{ I 21} & $\gamma_{2}$ & 23.8 & 17.24 & 0.85 & 17.29 & 0.91 & 17.24 & 0.87 \\
\hline & $\delta 1$ & 24.3 & 12.79 & 0.85 & 12.86 & 0.93 & & \\
\hline \multirow[t]{2}{*}{ I30 } & $\gamma 2$ & 10.4 & 17.96 & 0.82 & 23.37 & & 17.09 & -0.06 \\
\hline & $\delta 1$ & 12.0 & 13.35 & 0.64 & 15.00 & & 13.15 & 0.55 \\
\hline \multirow[t]{2}{*}{ I31 } & $\gamma 2$ & 11.9 & 18.45 & 0.75 & 28.85 & & 16.71 & -0.92 \\
\hline & $\delta 1$ & 9.8 & 14.65 & 0.34 & 33.74 & & 11.31 & -2.95 \\
\hline \multirow[t]{2}{*}{ I33 } & $\gamma 2$ & 10.6 & 17.24 & 0.71 & 8.89 & & 18.77 & 2.26 \\
\hline & $\delta 1$ & 12.2 & 12.24 & 0.08 & 9.89 & & 12.65 & 0.45 \\
\hline \multirow[t]{2}{*}{ I68 } & $\gamma 2$ & 11.4 & 19.25 & 0.79 & 28.19 & & 17.44 & -0.91 \\
\hline & $\delta 1$ & 8.4 & 13.87 & 0.56 & & & 9.07 & \\
\hline \multirow[t]{2}{*}{ I 90} & $\gamma 2$ & 15.6 & 18.42 & 0.41 & 15.93 & -2.28 & 18.93 & 0.92 \\
\hline & $\delta 1$ & 16.7 & 12.90 & 0.53 & 10.88 & -1.52 & & \\
\hline \multirow[t]{2}{*}{ I 97} & $\gamma 2$ & 9.1 & 18.43 & 1.01 & 8.34 & & 19.69 & 2.37 \\
\hline & $\delta 1$ & 11.4 & 14.99 & 0.88 & 9.50 & & 15.78 & 1.68 \\
\hline \multirow[t]{2}{*}{ I10 4} & $\gamma 2$ & 16.0 & 17.84 & 0.90 & 15.86 & -2.05 & 18.13 & 1.19 \\
\hline & $\delta 1$ & 14.4 & 10.34 & 0.71 & 7.50 & & 10.70 & 1.14 \\
\hline \multirow[t]{2}{*}{ I118 } & $\gamma^{2}$ & 22.6 & 15.96 & 0.66 & 15.47 & 0.17 & 16.02 & 0.75 \\
\hline & $\delta 1$ & 23.0 & 13.16 & 0.86 & 12.89 & 0.58 & 13.23 & 0.91 \\
\hline \multirow[t]{2}{*}{ I154 } & $\gamma^{2}$ & 13.7 & 16.96 & 0.76 & 23.43 & & 15.97 & -0.23 \\
\hline & $\delta 1$ & 16.3 & 11.78 & 0.73 & 17.48 & 6.40 & 10.94 & -0.16 \\
\hline \multirow[t]{2}{*}{ I170 } & $\gamma 2$ & 9.5 & 18.41 & 0.71 & 40.65 & & 15.06 & -2.55 \\
\hline & $\delta 1$ & 8.6 & 12.88 & 0.68 & 32.52 & & 10.26 & -1.98 \\
\hline \multirow[t]{2}{*}{ I193 } & $\gamma^{2}$ & & 17.46 & 0.81 & 17.55 & 0.91 & 17.42 & 0.78 \\
\hline & $\delta 1$ & & 13.04 & 0.83 & 13.15 & 0.94 & 13.00 & 0.80 \\
\hline
\end{tabular}

${ }^{a}$ Conditions: $25{ }^{\circ} \mathrm{C}, \mathrm{pH}$ 7.2. The chemical shifts in the cz- $\varepsilon 186 / \theta / \mathrm{La}^{3+}$ complex were measured from ${ }^{13} \mathrm{C}$-HSQC spectra of the sample containing ${ }^{13} \mathrm{C} /{ }^{15} \mathrm{~N}$ labeled cz- $\varepsilon 186$ in the presence of 1 equivalent $\mathrm{La}^{3+}$. Whenever possible, chemical shifts of the cz- $\varepsilon 186 / \theta / \mathrm{Dy}^{3+}$ and $\mathrm{cz}-\varepsilon 186 / \theta / \mathrm{Yb}^{3+}$ complexes were measured from ${ }^{13} \mathrm{C}-\mathrm{HSQC}$ spectra of samples prepared with 1:1 mixtures of $\mathrm{La}^{3+}$ and $\mathrm{Dy}^{3+}$, or $\mathrm{La}^{3+}$ and $\mathrm{Yb}^{3+}$, respectively. ${ }^{13} \mathrm{C}$ chemical shifts of methyl groups for which no ${ }^{1} \mathrm{H}$ chemical shift is reported were measured from the pd exchange peaks in $2 \mathrm{D}$ or $3 \mathrm{D}$ methyl $\mathrm{C}_{\mathrm{z}^{-}}$ EXSY spectra, whichever gave better resolution. When neither ${ }^{13} \mathrm{C}$ nor ${ }^{1} \mathrm{H}$ chemical shifts are indicated, the expected cross-peak could not be identified either because of spectral overlap (e.g. in the case of vanishing PCS) or strong PRE. 
Table S2. Number of correctly assigned methyl groups of Met, Thr, and Ala residues of cz- $\varepsilon 186$ using the program Possum. ${ }^{\text {a }}$

\begin{tabular}{|c|c|c|c|c|c|c|c|c|c|c|c|c|c|}
\hline \multicolumn{4}{|c|}{ Dy only } & \multicolumn{4}{|c|}{$\mathrm{Yb}$ only } & \multicolumn{6}{|c|}{ Dy and $\mathrm{Yb}$} \\
\hline 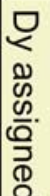 & 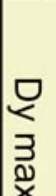 & 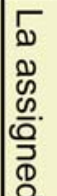 & 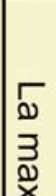 & 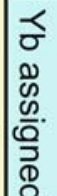 & 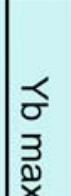 & 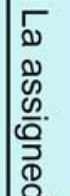 & 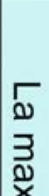 & 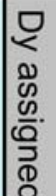 & 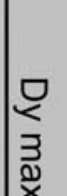 & 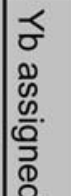 & 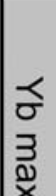 & 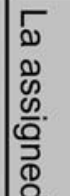 & 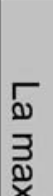 \\
\hline
\end{tabular}

\begin{tabular}{|c|c|c|c|c|c|c|c|c|c|c|c|c|c|c|c|}
\hline \multirow{5}{*}{ 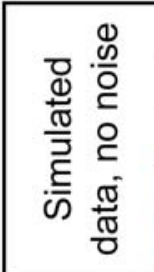 } & & 4 & 4 & 4 & 4 & 5 & 5 & 5 & 5 & 4 & 4 & 5 & 5 & 5 & \\
\hline & $T$ & 7 & 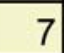 & 1 & 7 & 7 & 7 & 7 & 7 & 7 & 7 & 7 & 7 & 7 & 7 \\
\hline & A & 3 & 3 & 3 & 3 & 13 & 13 & 13 & 13 & 3 & 13 & 13 & 13 & 4 & 14 \\
\hline & Tot & 24 & 24 & 24 & 24 & 25 & 25 & 25 & 5 & 4 & 4 & 25 & 25 & 26 & 26 \\
\hline & $\%$ & \multicolumn{2}{|c|}{100.0} & \multicolumn{2}{|c|}{100.0} & \multicolumn{2}{|c|}{100.0} & \multicolumn{2}{|c|}{100.0} & \multicolumn{2}{|c|}{100.0} & \multicolumn{2}{|c|}{100.0} & \multicolumn{2}{|c|}{100.0} \\
\hline \multirow{5}{*}{ 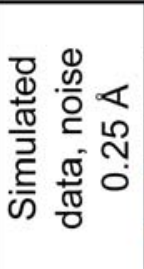 } & & 4 & 4 & 4 & 4 & 5 & 5 & 5 & 5 & 4 & 4 & 5 & $v$ & 5 & 5 \\
\hline & $\pi$ & I & 1 & I & 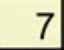 & 7 & 7 & 7 & 7 & 7 & & 7 & I & 7 & 7 \\
\hline & Ala & 1 & 13 & 6 & 13 & 12 & 13 & 10 & 13 & 12 & 13 & 12 & 13 & 11 & 14 \\
\hline & Tot: & 22 & 24 & 17 & 24 & 24 & 25 & 22 & 25 & 23 & 24 & 24 & 25 & 23 & 26 \\
\hline & $\%$ & \multicolumn{2}{|c|}{91.7} & \multicolumn{2}{|c|}{70.8} & \multicolumn{2}{|c|}{96.0} & \multicolumn{2}{|c|}{88.0} & \multicolumn{2}{|c|}{95.8} & \multicolumn{2}{|c|}{96.0} & \multicolumn{2}{|c|}{88.5} \\
\hline \multirow{5}{*}{ 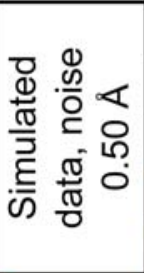 } & Met & 4 & 4 & 4 & 4 & 5 & 5 & 5 & 5 & 4 & 4 & 5 & 5 & 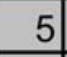 & 5 \\
\hline & $\mathrm{hr}$ & 7 & 7 & 7 & 7 & 7 & 7 & 7 & 7 & 7 & 1 & 7 & $t$ & 1 & 7 \\
\hline & Ala & 10 & 13 & 6 & 13 & 9 & 13 & 7 & 13 & 12 & 13 & 12 & 13 & 11 & 14 \\
\hline & Tot & 21 & 24 & 17 & 24 & 21 & 25 & 19 & 25 & 23 & 24 & 24 & 25 & 23 & 26 \\
\hline & 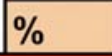 & \multicolumn{2}{|c|}{87.5} & \multicolumn{2}{|c|}{70.8} & \multicolumn{2}{|c|}{84.0} & \multicolumn{2}{|c|}{76.0} & \multicolumn{2}{|c|}{95.8} & \multicolumn{2}{|c|}{96.0} & \multicolumn{2}{|c|}{88.5} \\
\hline \multirow{5}{*}{ 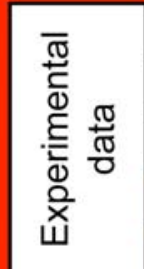 } & IV & 4 & 4 & 4 & 4 & 3 & 5 & 3 & 5 & 4 & 4 & 3 & 5 & 3 & 3 \\
\hline & Thr & 7 & 7 & 7 & 1 & 5 & 1 & 5 & 7 & 7 & 1 & 7 & 1 & 7 & $T$ \\
\hline & Ala & 8 & 13 & 6 & 13 & 11 & 13 & 11 & 13 & 11 & 13 & 13 & 13 & 14 & 14 \\
\hline & Tot & 19 & 24 & 17 & 24 & 19 & 25 & 19 & 25 & 22 & 24 & 23 & 25 & 24 & 26 \\
\hline & $\%$ & \multicolumn{2}{|c|}{79.2} & \multicolumn{2}{|c|}{70.8} & \multicolumn{2}{|c|}{76.0} & \multicolumn{2}{|c|}{76.0} & \multicolumn{2}{|c|}{91.7} & \multicolumn{2}{|c|}{92.0} & 92 & .3 \\
\hline
\end{tabular}

${ }^{a}$ Calculations were performed using the experimental data of Table S1 and simulated data, where the paramagnetic chemical shifts of Table S1 were replaced by chemical shifts back-calculated from the crystal structure of $\varepsilon 186$ and the $\Delta \chi$ tensors used in the present study. Two additional sets of simulated data were generated by addition of structural noise to the PDB coordinates of $\varepsilon 186$. The structural noise followed a Gaussian distribution of 0.25 and $0.5 \AA$ standard deviation, resulting in a Maxwell-Boltzmann distribution of atomic displacements with maxima at 0.35 and $0.7 \AA$, respectively. The columns marked "Dy max", "Yb max", and "La max" report the number of methyl groups for which data in the paramagnetic state were available to the program. (Additional peaks observed in the diamagnetic state remained unassigned.) The results are reported for calculations where the diamagnetic chemical shifts were supplemented only with data from $\mathrm{Dy}^{3+}$ (light yellow), $\mathrm{Yb}^{3+}$ (light blue) or both (grey). The rows marked with the \% symbol display the percentage of correctly assigned methyl groups for all three residues. The program Possum is available from http://compbio.chemistry.uq.edu.au/bmmg/christophe. 
Table S3. Number of correctly assigned methyl groups of Val, Leu, and Ile residues of cz- $\varepsilon 186$ using the program Possum with methyl connectivity information in the $\mathrm{Yb}^{3+}$ complex. ${ }^{\mathrm{a}}$
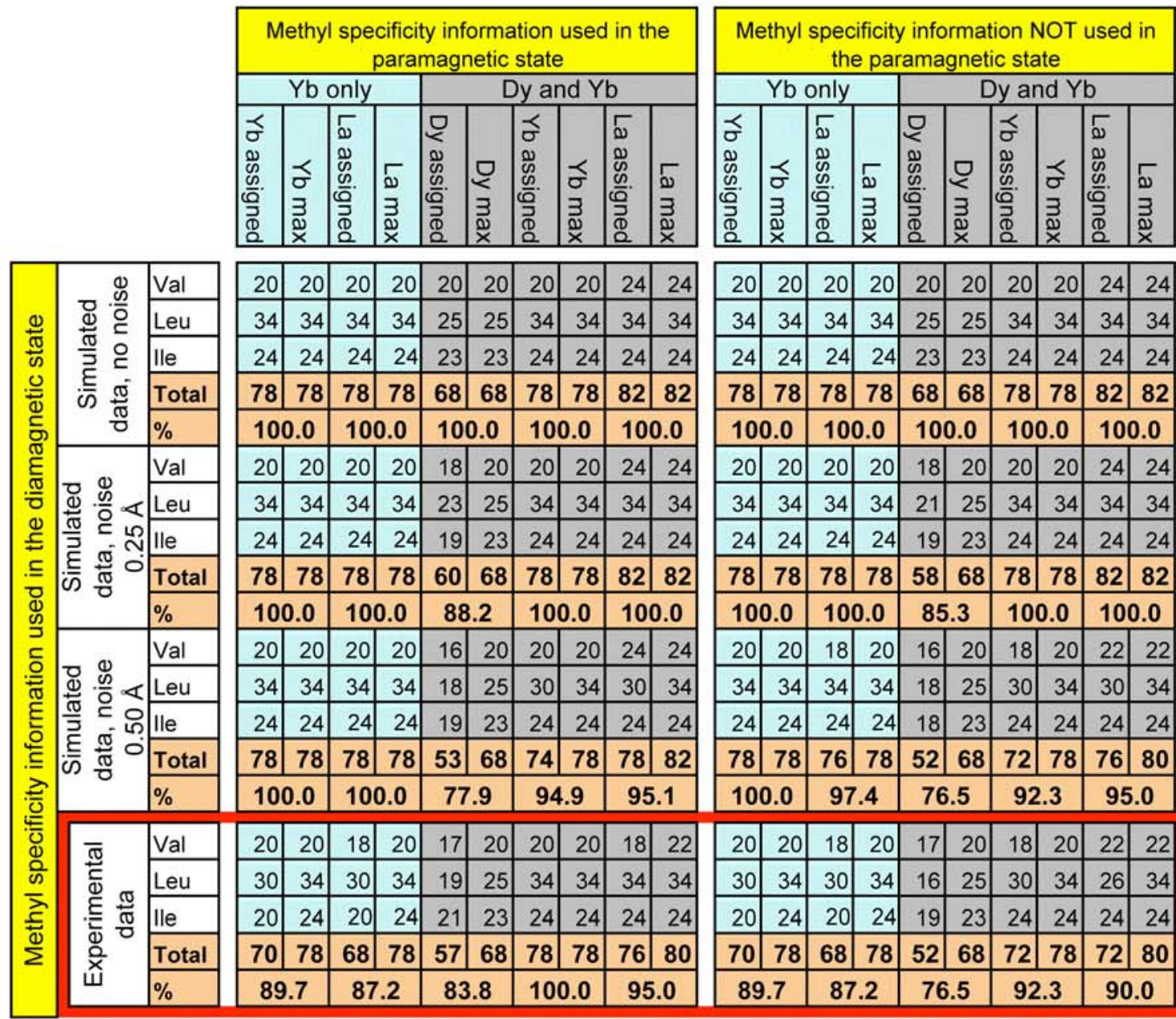

\begin{tabular}{|c|c|c|c|c|c|c|c|c|c|}
\hline 20 & 20 & 20 & 20 & 20 & 20 & 20 & 20 & 24 & 24 \\
\hline 34 & 34 & 34 & 34 & 25 & 25 & 34 & 34 & 34 & 34 \\
\hline 24 & 24 & 24 & 24 & 23 & 23 & 24 & 24 & 24 & 24 \\
\hline $\mathbf{7 8}$ & $\mathbf{7 8}$ & $\mathbf{7 8}$ & $\mathbf{7 8}$ & $\mathbf{6 8}$ & $\mathbf{6 8}$ & $\mathbf{7 8}$ & $\mathbf{7 8}$ & $\mathbf{8 2}$ & $\mathbf{8 2}$ \\
\hline $\mathbf{1 0 0 . 0}$ & $\mathbf{1 0 0 . 0}$ & $\mathbf{1 0 0 . 0}$ & $\mathbf{1 0 0 . 0}$ & $\mathbf{1 0 0 . 0}$ \\
\hline 20 & 20 & 20 & 20 & 18 & 20 & 20 & 20 & 24 & 24 \\
\hline 34 & 34 & 34 & 34 & 21 & 25 & 34 & 34 & 34 & 34 \\
\hline 24 & 24 & 24 & 24 & 19 & 23 & 24 & 24 & 24 & 24 \\
\hline $\mathbf{7 8}$ & $\mathbf{7 8}$ & $\mathbf{7 8}$ & $\mathbf{7 8}$ & $\mathbf{5 8}$ & $\mathbf{6 8}$ & $\mathbf{7 8}$ & $\mathbf{7 8}$ & $\mathbf{8 2}$ & $\mathbf{8 2}$ \\
\hline $\mathbf{1 0 0 . 0}$ & $\mathbf{1 0 0 . 0}$ & $\mathbf{8 5 . 3}$ & $\mathbf{1 0 0 . 0}$ & $\mathbf{1 0 0 . 0}$ \\
\hline 20 & 20 & 18 & 20 & 16 & 20 & 18 & 20 & 22 & 22 \\
\hline 34 & 34 & 34 & 34 & 18 & 25 & 30 & 34 & 30 & 34 \\
\hline 24 & 24 & 24 & 24 & 18 & 23 & 24 & 24 & 24 & 24 \\
\hline $\mathbf{7 8}$ & $\mathbf{7 8}$ & $\mathbf{7 6}$ & $\mathbf{7 8}$ & $\mathbf{5 2}$ & $\mathbf{6 8}$ & $\mathbf{7 2}$ & $\mathbf{7 8}$ & $\mathbf{7 6}$ & $\mathbf{8 0}$ \\
\hline $\mathbf{1 0 0 . 0}$ & $\mathbf{9 7 . 4}$ & $\mathbf{7 6 . 5}$ & $\mathbf{9 2 . 3}$ & $\mathbf{9 5 . 0}$ \\
\hline 20 & 20 & 18 & 20 & 17 & 20 & 18 & 20 & 22 & 22 \\
\hline 30 & 34 & 30 & 34 & 16 & 25 & 30 & 34 & 26 & 34 \\
\hline 20 & 24 & 20 & 24 & 19 & 23 & 24 & 24 & 24 & 24 \\
\hline $\mathbf{7 0}$ & $\mathbf{7 8}$ & $\mathbf{6 8}$ & $\mathbf{7 8}$ & $\mathbf{5 2}$ & $\mathbf{6 8}$ & $\mathbf{7 2}$ & $\mathbf{7 8}$ & $\mathbf{7 2}$ & $\mathbf{8 0}$ \\
\hline $\mathbf{8 9 . 7}$ & $\mathbf{8 7 . 2}$ & $\mathbf{7 6 . 5}$ & $\mathbf{9 2 . 3}$ & $\mathbf{9 0 . 0}$ \\
\hline
\end{tabular}

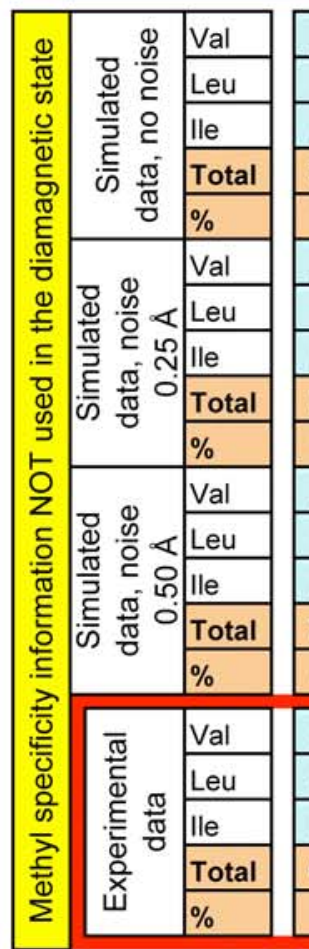

20

\begin{tabular}{|l|l|l|l|l|l|l|l|l|l|}
\hline 20 & 20 & 20 & 20 & 20 & 20 & 20 & 20 & 24 & 24 \\
\hline
\end{tabular} \begin{tabular}{lllllllllllll}
34 & 34 & 34 & 34 & 25 & 25 & 34 & 34 & 34 & 34 \\
\hline
\end{tabular} \begin{tabular}{|l|lllllllllll|}
\hline 24 & 24 & 24 & 24 & 23 & 23 & 24 & 24 & 24 & 24 \\
\hline
\end{tabular}

\begin{tabular}{|l|l|l|l|l|l|l|l|l|l|}
\hline 78 & 78 & 78 & 78 & 68 & 68 & 78 & 78 & 82 & 82 \\
\hline
\end{tabular} \begin{tabular}{|l|l|l|l|l|l|}
\hline 100.0 & 100.0 & 100.0 & 100.0 & 100.0 \\
\hline
\end{tabular} \begin{tabular}{|l|l|l|l|l|l|l|l|l|l|}
\hline 20 & 20 & 18 & 20 & 20 & 20 & 20 & 20 & 20 & 24 \\
\hline
\end{tabular} \begin{tabular}{llllllllllll}
34 & 34 & 34 & 34 & 25 & 25 & 34 & 34 & 34 & 34 \\
\hline
\end{tabular} \begin{tabular}{llllllllllll}
24 & 24 & 24 & 24 & 23 & 23 & 24 & 24 & 24 & 24 \\
\hline
\end{tabular} \begin{tabular}{|l|l|l|l|l|l|l|l|l|l|}
\hline 78 & 78 & 78 & 78 & 68 & 68 & 78 & 78 & 82 & 82 \\
\hline
\end{tabular} \begin{tabular}{|c|c|c|c|c|}
\hline 100.0 & 100.0 & 100.0 & 100.0 & 100.0 \\
\hline
\end{tabular} \begin{tabular}{|l|l|l|l|l|l|l|l|l|l|}
\hline 20 & 20 & 18 & 20 & 18 & 20 & 20 & 20 & 24 & 24 \\
\hline 34 & 34 & 34 & 34 & 2 & 25 & 34 & 34 & 34 & 34 \\
\hline
\end{tabular} \begin{tabular}{lllllllllllll|}
34 & 34 & 34 & 34 & 22 & 25 & 34 & 34 & 34 & 34 \\
\hline
\end{tabular} \begin{tabular}{|l|l|l|l|l|l|l|l|l|l|}
34 & 34 & 34 & 34 & 20 & 25 & 34 & 34 & 34 & 34 \\
\hline
\end{tabular}

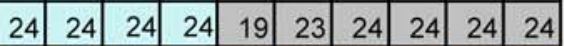
\begin{tabular}{|c|c|c|c|c|c|c|c|c|c|}
\hline 78 & 78 & 76 & 78 & 61 & 68 & 78 & 78 & 78 & 82 \\
\hline
\end{tabular} \begin{tabular}{|c|c|c|c|c|}
\hline 100.0 & 97.4 & 89.7 & 100.0 & 95.1 \\
\hline
\end{tabular} \begin{tabular}{l|l|l|l|l|l|l|l|l|l|}
24 & 24 & 24 & 24 & 19 & 23 & 24 & 24 & 24 & 24 \\
\hline
\end{tabular} \begin{tabular}{|l|l|l|l|l|l|l|l|l|l|}
\hline 78 & 78 & 76 & 78 & 57 & 68 & 78 & 78 & 82 & 82 \\
\hline
\end{tabular} \begin{tabular}{|c|c|c|c|c|}
\hline 100.0 & 97.4 & 83.8 & 100.0 & 100.0 \\
\hline
\end{tabular} \begin{tabular}{|l|l|l|l|l|l|l|l|l|l|}
20 & 20 & 18 & 20 & 14 & 20 & 20 & 20 & 18 & 24 \\
\hline
\end{tabular} \begin{tabular}{|l|l|l|l|l|l|l|l|l|l|}
\hline 20 & 20 & 18 & 20 & 14 & 20 & 20 & 20 & 18 & 24 \\
\hline
\end{tabular} \begin{tabular}{lllllllllllll}
34 & 34 & 34 & 34 & 18 & 25 & 30 & 34 & 30 & 34 \\
\hline
\end{tabular} \begin{tabular}{llllllllllllll}
34 & 34 & 34 & 34 & 17 & 25 & 30 & 34 & 30 & 34 \\
\hline
\end{tabular} \begin{tabular}{|l|l|l|l|l|l|l|l|l|l|}
24 & 24 & 24 & 24 & 18 & 23 & 24 & 24 & 24 & 24 \\
\hline
\end{tabular} \begin{tabular}{|l|l|l|l|l|l|l|l|l|l|}
78 & 78 & 76 & 78 & 50 & 68 & 74 & 78 & 72 & 82 \\
\hline
\end{tabular} \begin{tabular}{lllllllllll|l}
20 & 24 & 20 & 24 & 17 & 23 & 20 & 24 & 20 & 24 \\
\hline
\end{tabular} \begin{tabular}{|c|c|c|c|c|c|}
\hline 100.0 & 97.4 & 73.5 & 94.9 & 87.8 \\
\hline
\end{tabular} \begin{tabular}{|l|l|l|l|l|l|l|l|l|l|}
74 & 78 & 72 & 78 & 48 & 68 & 70 & 78 & 68 & 82 \\
\hline
\end{tabular} \begin{tabular}{|l|l|l|l|l|l|}
\hline 94.9 & 92.3 & 70.6 & 89.7 & 82.9 \\
\hline
\end{tabular}

\begin{tabular}{|l|l|l|l|l|l|l|l|l|l|l|l|l|l|l|l|l|l|l|l|}
\hline 20 & 20 & 18 & 20 & 17 & 20 & 20 & 20 & 20 & 22 & 20 & 20 & 18 & 20 & 17 & 20 & 20 & 20 & 18 & 22 \\
\hline
\end{tabular} \begin{tabular}{llllllllllllll}
26 & 34 & 24 & 34 & 15 & 25 & 30 & 34 & 26 & 34 \\
\hline
\end{tabular} \begin{tabular}{llllllllllll}
26 & 34 & 26 & 34 & 11 & 25 & 26 & 34 & 26 & 34 \\
\hline
\end{tabular} \begin{tabular}{llllllllllll|}
20 & 24 & 20 & 24 & 21 & 23 & 24 & 24 & 24 & 24 \\
\hline
\end{tabular} \begin{tabular}{lllllllllllll}
20 & 24 & 20 & 24 & 19 & 23 & 24 & 24 & 24 & 24 \\
\hline
\end{tabular} \begin{tabular}{|l|l|l|l|l|l|l|l|l|l|}
66 & 78 & 62 & 78 & 53 & 68 & 74 & 78 & 70 & 80 \\
\hline
\end{tabular} \begin{tabular}{|l|l|l|l|l|l|l|l|l|l|}
66 & 78 & 64 & 78 & 47 & 68 & 70 & 78 & 68 & 80 \\
\hline
\end{tabular} \begin{tabular}{|c|c|c|c|c|}
\hline 84.6 & 79.5 & 77.9 & 94.9 & 87.5 \\
\hline
\end{tabular} \begin{tabular}{|l|l|l|l|l|}
\hline 84.6 & 82.1 & 69.1 & 89.7 & 85.0 \\
\hline
\end{tabular} 
a Calculations were performed using the experimental data of Table S1 and simulated data as described in the footnote of Table S2. As each Val, Leu and Ile residue contains two methyl groups, methyl specificity and methyl connectivity information can be used as additional information to support the resonance assignment. (Methyl specificity information refers to stereospecific assignments of the methyl groups of Val and Leu and the a priori distinction of $\gamma_{2}$ and $\delta_{1}$ methyl groups of Ile. Methyl connectivity information refers to the knowledge of which peaks arise from the same residue.) The results of four different combinations are shown, with and without methyl specificity information in the paramagnetic complexes, and with and without methyl specificity information in the diamagnetic complex. It was assumed that no methyl connectivity information can be established for the $\mathrm{Dy}^{3+}$ complex because of strong PRE. The data are presented in the same format as in Table S2. Assignments were counted as correct whenever a methyl cross-peak was assigned to the correct residue, disregarding the stereospecific correctness of the assignment. Note that the maximum number of assignable methyl groups reported in the column marked "La max" can vary when both $\mathrm{Dy}^{3+}$ and $\mathrm{Yb}^{3+}$ data are used, because Possum has the freedom not to assign every HSQC cross-peak observed for the $\mathrm{Dy}^{3+}$ complex to a peak observed for the $\mathrm{Yb}^{3+}$ complex. This results in a small variation of the number of residues for which the program has paramagnetic information available and can attempt an assignment of the diamagnetic data. 
Table S4. Number of correctly assigned methyl groups of valine, leucine, and isoleucine residues of cz-E186 using the program Possum without methyl connectivity information in the $\mathrm{Yb}^{3+}$ complex. ${ }^{\text {a }}$

\begin{tabular}{|c|c|c|c|c|c|c|c|c|c|c|c|c|c|}
\hline \multicolumn{14}{|c|}{ Methyl specificity information used in the paramagnetic state } \\
\hline \multicolumn{4}{|c|}{ Dy only } & \multicolumn{4}{|c|}{ Yb only } & \multicolumn{6}{|c|}{ Dy and $\mathrm{Yb}$} \\
\hline $\begin{array}{l}u_{0} \\
\end{array}$ & 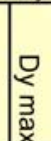 & 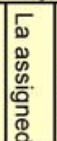 & 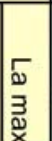 & 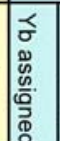 & $\begin{array}{l}\sigma \\
\sigma \\
\bar{z} \\
\mathbb{\alpha}\end{array}$ & 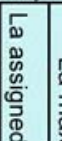 & 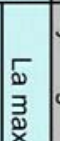 & 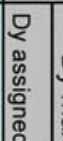 & & 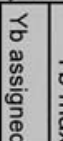 & 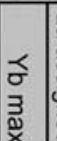 & 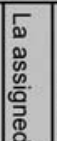 & 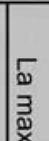 \\
\hline
\end{tabular}

\begin{tabular}{|c|c|c|c|c|c|c|c|c|c|c|c|c|}
\hline \multicolumn{13}{|c|}{$\begin{array}{l}\text { Methyl specificity information NOT used in the paramagnetic } \\
\text { state }\end{array}$} \\
\hline \multicolumn{4}{|c|}{ Dy only } & \multicolumn{3}{|c|}{ Yb only } & \multicolumn{6}{|c|}{ Dy and $\mathrm{Yb}$} \\
\hline & 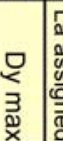 & 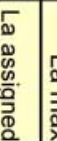 & 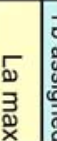 & 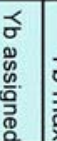 & 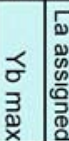 & 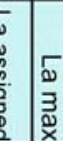 & 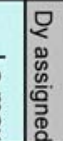 & 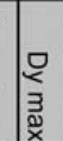 & 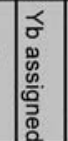 & 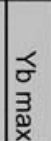 & 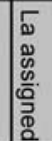 & 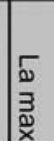 \\
\hline
\end{tabular}

\begin{tabular}{|c|c|c|c|c|c|c|c|c|c|c|c|c|c|c|c|c|}
\hline \multirow{5}{*}{ 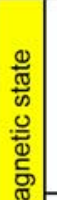 } & \multirow{5}{*}{ 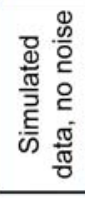 } & Val & 20 & 20 & 22 & 22 & 19 & 19 & 20 & 20 & 20 & 20 & 19 & 19 & 24 & 24 \\
\hline & & Leu & 25 & 25 & 28 & 28 & 33 & 33 & 34 & 34 & 25 & 25 & 33 & 33 & 34 & 34 \\
\hline & & lle & 23 & 23 & 24 & 24 & 22 & 22 & 24 & 24 & 23 & 23 & 22 & 22 & 24 & 24 \\
\hline & & Total & 68 & 68 & 74 & 74 & 74 & 74 & 78 & 78 & 68 & 68 & 74 & 74 & 82 & 82 \\
\hline & & $\%$ & \multicolumn{2}{|c|}{100.0} & \multicolumn{2}{|c|}{100.0} & \multicolumn{2}{|c|}{100.0} & \multicolumn{2}{|c|}{100.0} & \multicolumn{2}{|c|}{100.0} & \multicolumn{2}{|c|}{100.0} & \multicolumn{2}{|c|}{100.0} \\
\hline & \multirow{5}{*}{ 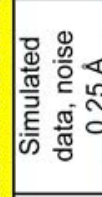 } & Val & 18 & 20 & 20 & 22 & 19 & 19 & 20 & 20 & 18 & 20 & 19 & 19 & 24 & 24 \\
\hline & & Leu & 21 & 25 & 24 & 30 & 33 & 33 & 34 & 34 & 22 & 25 & 33 & 33 & 34 & 34 \\
\hline & & |lle & 22 & 23 & 14 & 24 & 22 & 22 & 24 & 24 & 19 & 23 & 22 & 22 & 24 & 24 \\
\hline & & Total & 61 & 68 & 58 & 76 & 74 & 74 & 78 & 78 & 59 & 68 & 74 & 74 & 82 & 82 \\
\hline & & $\%$ & \multicolumn{2}{|c|}{89.7} & \multicolumn{2}{|c|}{76.3} & \multicolumn{2}{|c|}{100.0} & \multicolumn{2}{|c|}{100.0} & \multicolumn{2}{|c|}{86.8} & \multicolumn{2}{|c|}{100.0} & \multicolumn{2}{|c|}{100.0} \\
\hline & \multirow{5}{*}{ 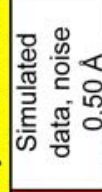 } & Val & 16 & 20 & 20 & 22 & 19 & 19 & 20 & 20 & 16 & 20 & 18 & 19 & 24 & 24 \\
\hline & & Leu & 14 & 25 & 14 & 30 & 33 & 33 & 34 & 34 & 18 & 25 & \begin{tabular}{|l|}
29 \\
\end{tabular} & 33 & 30 & 34 \\
\hline & & |lle & 16 & 23 & 6 & 24 & 22 & 22 & 24 & 24 & 19 & 23 & 22 & 22 & 24 & 24 \\
\hline & & Total & 46 & 68 & 40 & 76 & 74 & 74 & 78 & 78 & 53 & 68 & 69 & 74 & 78 & 82 \\
\hline & & $\%$ & \multicolumn{2}{|c|}{67.6} & \multicolumn{2}{|c|}{52.6} & \multicolumn{2}{|c|}{100.0} & \multicolumn{2}{|c|}{100.0} & \multicolumn{2}{|c|}{77.9} & \multicolumn{2}{|c|}{93.2} & \multicolumn{2}{|c|}{95.1} \\
\hline & \multirow{5}{*}{ 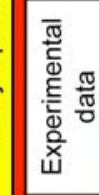 } & Val & 17 & 20 & 20 & 20 & 18 & 19 & 20 & 22 & 17 & 20 & 18 & 19 & 20 & 24 \\
\hline & & Leu & 14 & 25 & 10 & 30 & 25 & 33 & 24 & 34 & 19 & 25 & 33 & 33 & 34 & 34 \\
\hline & & lle & 19 & 23 & 18 & 24 & 19 & 22 & 20 & 24 & 21 & 23 & 22 & 22 & 24 & 24 \\
\hline & & Total & 50 & 68 & 48 & 74 & 62 & 74 & 64 & 80 & 57 & 68 & 73 & 74 & 78 & 82 \\
\hline & & $\%$ & 73 & & 64 & & 83 & 3.8 & 80 & & 83 & & 98 & & 95 & 5.1 \\
\hline
\end{tabular}

\begin{tabular}{|l|l|l|l|l|l|l|l|l|l|l|l|l|l|}
\hline 20 & 20 & 22 & 22 & 19 & 19 & 20 & 20 & 20 & 20 & 19 & 19 & 24 & 24 \\
\hline & 2 & 2 & 2 & 33 & 33 & 34 & 34 & 2 & 25 & 33 & 33 & 34 & \\
\hline
\end{tabular} \begin{tabular}{l|l|l|l|l|l|l|l|l|l|l|l|l|l}
\hline 25 & 25 & 26 & 28 & 33 & 33 & 34 & 34 & 25 & 25 & 33 & 33 & 34 & 34 \\
\hline
\end{tabular} \begin{tabular}{lllllllllllllllllll}
\hline 23 & 23 & 24 & 24 & 22 & 22 & 24 & 24 & 23 & 23 & 22 & 22 & 24 & 24 \\
\hline
\end{tabular}

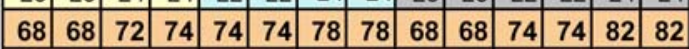
\begin{tabular}{|l|l|l|l|l|l|l|}
\hline 100.0 & 97.3 & 100.0 & 100.0 & 100.0 & 100.0 & 100.0 \\
\hline
\end{tabular} \begin{tabular}{|l|ll|ll|ll|l|l|l|l|l|l|l|}
\hline 18 & 20 & 20 & 22 & 19 & 19 & 20 & 20 & 18 & 20 & 19 & 19 & 24 & 24 \\
\hline
\end{tabular} \begin{tabular}{lllllllllllllll|}
\hline 21 & 25 & 18 & 30 & 33 & 33 & 34 & 34 & 20 & 25 & 33 & 33 & 34 & 34 \\
\hline
\end{tabular} \begin{tabular}{|llllllllllllllll|}
\hline 18 & 23 & 12 & 24 & 22 & 22 & 24 & 24 & 19 & 23 & 20 & 22 & 24 & 24 \\
\hline
\end{tabular} \begin{tabular}{|c|c|c|c|c|c|c|c|c|c|c|c|c|c|}
\hline 57 & 68 & 50 & 76 & 74 & 74 & 78 & 78 & 57 & 68 & 72 & 74 & 82 & 82 \\
\hline
\end{tabular} \begin{tabular}{|c|c|c|c|c|c|c|c|}
\hline 83.8 & 65.8 & 100.0 & 100.0 & 83.8 & 97.3 & 100.0 \\
\hline
\end{tabular} \begin{tabular}{|l|l|l|l|l|l|l|l|l|l|l|l|l|l|}
\hline 13 & 20 & 16 & 22 & 18 & 19 & 18 & 22 & 15 & 20 & 18 & 19 & 20 & 24 \\
\hline & & & & 2 & &
\end{tabular} \begin{tabular}{|l|l|l|l|l|l|l|l|l|l|l|l|l|l|}
\hline 9 & 25 & 8 & 28 & 27 & 33 & 24 & 34 & 15 & 25 & 25 & 33 & 24 & 34 \\
\hline
\end{tabular}

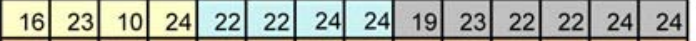
\begin{tabular}{|l|l|l|l|l|l|l|l|l|l|l|l|l|l|}
\hline 38 & 68 & 34 & 74 & 67 & 74 & 66 & 80 & 49 & 68 & 65 & 74 & 68 & 82 \\
\hline
\end{tabular} \begin{tabular}{|c|c|c|c|c|c|c|}
\hline 55.9 & 45.9 & 90.5 & 82.5 & 72.1 & 87.8 & 82.9 \\
\hline
\end{tabular}

\begin{tabular}{|c|c|c|c|c|c|c|c|c|c|c|c|c|c|c|}
\hline \multirow{4}{*}{ 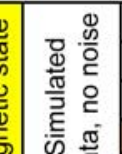 } & & 0 & & & & 19 & & & & & & & & \\
\hline & eu & & & 26 & & 33 & & 34 & & 25 & & 33 & & \\
\hline & II & & & 4 & & 22 & & 24 & & 23 & & 22 & & \\
\hline & Total & & 68 & 7 & 4 & $\begin{array}{ll}4 & 7 \\
\end{array}$ & 4 & $\begin{array}{ll}87 \\
\end{array}$ & 18 & \begin{tabular}{l|l}
68 \\
\end{tabular} & 68 & \begin{tabular}{ll|l}
774 & 7 \\
\end{tabular} & & \\
\hline \multirow[t]{2}{*}{$\frac{\pi}{0}$} & 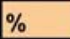 & 100. & & 97.3 & & 100. & & 100. & & 100. & & 100.0 & & 100.0 \\
\hline & |al & 18 & 2 & 20 & 24 & 19 & 19 & 18 & 20 & 20 & 20 & 19 & & \\
\hline \multirow{4}{*}{ 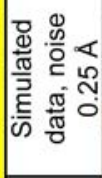 } & Leu & 6 & 25 & 16 & 30 & 33 & 33 & 34 & 34 & 22 & 25 & \begin{tabular}{l|l}
33 & 3 \\
\end{tabular} & & \\
\hline & Ile & 2. & 23 & 14 & 24 & 22 & 22 & 24 & 24 & 19 & 23 & 22 & 2 & 24 \\
\hline & Total & \begin{tabular}{l|l}
56 \\
\end{tabular} & 68 & \begin{tabular}{l|l}
50 & 7 \\
\end{tabular} & 8 & \begin{tabular}{l|l}
774 & 7
\end{tabular} & 4 & \begin{tabular}{l|l}
76 &
\end{tabular} & 78 & \begin{tabular}{l|l}
61 & 6
\end{tabular} & 68 & \begin{tabular}{|l|l}
74 & 7 \\
\end{tabular} & & 88 \\
\hline & $\%$ & 82.4 & & 64.1 & & 100. & & 97.4 & & 89.7 & & 100.0 & & 95.1 \\
\hline \multirow{5}{*}{ 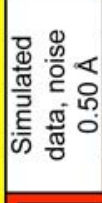 } & $\mathrm{Val}$ & 17 & 20 & \begin{tabular}{l|l}
16 & 2
\end{tabular} & 22 & 15 & 19 & 14 & 22 & 14 & 20 & 17 & & \\
\hline & Leu & 14 & 25 & 14 & 30 & 31 & 33 & 28 & 34 & 13 & $\underline{25}$ & $\begin{array}{ll}27 & 3 \\
\end{array}$ & & 24 \\
\hline & Ile & 17 & 23 & 의 & 24 & 22 & 22 & 24 & 24 & 18 & 23 & 22 & 2 & \\
\hline & Total & & 68 & \begin{tabular}{l|l}
36 & 7
\end{tabular} & 6 & \begin{tabular}{l|l}
68 & 7
\end{tabular} & 44 & \begin{tabular}{l|l}
66 & 8
\end{tabular} & 30 & $\begin{array}{lll}45 & 6\end{array}$ & 68 & \begin{tabular}{l|l}
66 & 7 \\
\end{tabular} & 4 & \begin{tabular}{l|l}
56 & 8
\end{tabular} \\
\hline & $\%$ & 70.6 & & 47.4 & & 91.9 & & 82.5 & & 66.2 & & 89.2 & & 82.5 \\
\hline \multirow{5}{*}{ 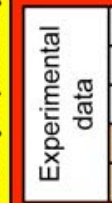 } & Val & 17 & 20 & 20 & 22 & 19 & 19 & 18 & 20 & 17 & 20 & \begin{tabular}{l|l}
19 & 1
\end{tabular} & & \\
\hline & Leu & 15 & 25 & 10 & 30 & 26 & 33 & 20 & 34 & 11 & 25 & \begin{tabular}{l|l}
26 & 3 \\
\end{tabular} & 2 & \\
\hline & lle & & 23 & 18 & 24 & 19 & 22 & 20 & 24 & 21 & 23 & \begin{tabular}{l|l}
22 & 2 \\
\end{tabular} & 2 & 24 \\
\hline & Total & \begin{tabular}{l|l}
50 \\
\end{tabular} & 68 & \begin{tabular}{l|l}
48 & 7 \\
\end{tabular} & 6 & \begin{tabular}{l|l}
64 & 7 \\
\end{tabular} & 44 & \begin{tabular}{l|l}
58 & 7 \\
\end{tabular} & 78 & 49 & 68 & \begin{tabular}{l|l}
67 & 7 \\
\end{tabular} & 6 & \begin{tabular}{l|l}
22 & 8 \\
\end{tabular} \\
\hline & & 73.5 & & 63.2 & & & & & & & & & & 77.5 \\
\hline
\end{tabular}

\begin{tabular}{|c|c|c|c|c|c|c|c|c|c|c|c|c|c|}
\hline 17 & 20 & 20 & 20 & 18 & 19 & 22 & 22 & 17 & 20 & 18 & 19 & 24 & 24 \\
\hline 12 & 25 & 12 & 30 & 25 & 33 & 24 & 34 & 16 & 25 & 29 & 33 & 26 & 34 \\
\hline 17 & 23 & 14 & 24 & 19 & 22 & 20 & 24 & 17 & 23 & 22 & 22 & 24 & 24 \\
\hline $\mathbf{4 6}$ & $\mathbf{6 8}$ & $\mathbf{4 6}$ & $\mathbf{7 4}$ & $\mathbf{6 2}$ & $\mathbf{7 4}$ & $\mathbf{6 6}$ & $\mathbf{8 0}$ & $\mathbf{5 0}$ & $\mathbf{6 8}$ & $\mathbf{6 9}$ & $\mathbf{7 4}$ & $\mathbf{7 4}$ & $\mathbf{8 2}$ \\
\hline $\mathbf{6 7 . 6}$ & $\mathbf{6 2 . 2}$ & $\mathbf{8 3 . 8}$ & $\mathbf{8 2 . 5}$ & $\mathbf{7 3 . 5}$ & $\mathbf{9 3 . 2}$ & $\mathbf{9 0 . 2}$ \\
\hline
\end{tabular}

\begin{tabular}{|l|l|l|l|l|l|l|l|l|l|l|l|l|l|}
\hline 20 & 20 & 22 & 22 & 19 & 19 & 20 & 20 & 20 & 20 & 19 & 19 & 24 & 24 \\
\hline
\end{tabular} \begin{tabular}{lllllllllllllll|}
\hline 25 & 25 & 26 & 28 & 33 & 33 & 34 & 34 & 25 & 25 & 33 & 33 & 34 & 34 \\
\hline
\end{tabular} \begin{tabular}{|llllllllllllllll}
\hline 23 & 23 & 24 & 24 & 22 & 22 & 24 & 24 & 23 & 23 & 22 & 22 & 24 & 24 \\
\hline
\end{tabular} \begin{tabular}{|l|l|l|l|l|l|l|l|l|l|l|l|l|l|}
\hline 68 & 68 & 72 & 74 & 74 & 74 & 78 & 78 & 68 & 68 & 74 & 74 & 82 & 82 \\
\hline
\end{tabular} \begin{tabular}{|l|c|c|c|c|c|c|}
\hline 100.0 & 97.3 & 100.0 & 100.0 & 100.0 & 100.0 & 100.0 \\
\hline
\end{tabular}

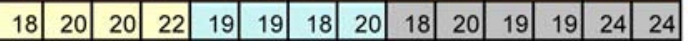
\begin{tabular}{llllllllllllllllll}
\hline 13 & 25 & 14 & 30 & 33 & 33 & 34 & 34 & 20 & 25 & 33 & 33 & 34 & 34 \\
\hline
\end{tabular} \begin{tabular}{|l|l|l|l|l|l|lllllllll}
\hline 18 & 23 & 12 & 24 & 22 & 22 & 24 & 24 & 17 & 23 & 21 & 22 & 24 & 24 \\
\hline 49 & 68 & 46 & 76 & 74 & 74 & & 78 & 55 & 68 & 73 & 74 & 82 & 82 \\
\hline
\end{tabular} \begin{tabular}{|l|l|l|l|l|l|l|l|l|l|l|l|l|l|}
\hline 49 & 68 & 46 & 76 & 74 & 74 & 76 & 78 & 55 & 68 & 73 & 74 & 82 & 82 \\
\hline
\end{tabular} \begin{tabular}{|c|c|c|c|c|c|c|}
\hline 72.1 & 60.5 & 100.0 & 97.4 & 80.9 & 98.6 & 100.0 \\
\hline
\end{tabular}

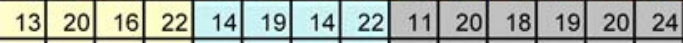
\begin{tabular}{|l|l|l|l|l|l|llllllllll}
\hline 12 & 25 & 10 & 30 & 27 & 33 & 26 & 34 & 15 & 25 & 24 & 33 & 24 & 34 \\
\hline
\end{tabular} \begin{tabular}{|l|l|l|l|l|l|l|l|l|l|l|l|l|l|}
\hline 16 & 23 & 6 & 24 & 22 & 22 & 20 & 24 & 16 & 23 & 18 & 22 & 20 & 24 \\
\hline 41 & 68 & 32 & 7 & 63 & 74 & 60 & 80 & 42 & 68 & 60 & 74 & 64 & 82 \\
\hline
\end{tabular} \begin{tabular}{|l|l|l|l|l|l|l|l|l|l|l|l|l|l|}
\hline 41 & 68 & 32 & 76 & 63 & 74 & 60 & 80 & 42 & 68 & 60 & 74 & 64 & 82 \\
\hline
\end{tabular} \begin{tabular}{|l|c|c|c|c|c|c|}
\hline 60.3 & 42.1 & 85.1 & 75.0 & 61.8 & 81.1 & 78.0 \\
\hline
\end{tabular} \begin{tabular}{|l|l|l|l|l|l|l|l|l|l|l|l|l|l|}
\hline 16 & 20 & 20 & 22 & 18 & 19 & 18 & 22 & 16 & 20 & 18 & 19 & 20 & 24 \\
\hline & 2 & 25 & & 32 & 22 & 33 & & &
\end{tabular} \begin{tabular}{|lllllllllllllllll}
12 & 25 & 12 & 30 & 22 & 33 & 18 & 34 & 11 & 25 & 25 & 33 & 20 & 34 \\
\hline
\end{tabular}

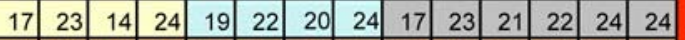
\begin{tabular}{|c|c|c|c|c|c|c|c|c|c|c|c|c|c|}
\hline 45 & 68 & 46 & 76 & 59 & 74 & 56 & 80 & 44 & 68 & 64 & 74 & 64 & 82 \\
\hline
\end{tabular} \begin{tabular}{|l|c|c|c|c|c|c|c|}
\hline 66.2 & 60.5 & 79.7 & 70.0 & 64.7 & 86.5 & 78.0 \\
\hline
\end{tabular}

${ }^{a}$ The data are presented as in Table S3. 